of the study was the small numbers of the patients in the single group and the early disease stage.Further studies are needed to analyze the validity and reproducibility of these biomarkers in early axSpA.

Disclosure of Interests: None declared

DOI: 10.1136/annrheumdis-2019-eular.2621

\section{AB1328 MEASUREMENT PROPERTIES OF THE BRIEF PAIN INVENTORY-SHORT FORM (BPI-SF) AND THE REVISED SHORT-FORM MCGILL PAIN QUESTIONNAIRE- VERSION-2 (SF-MPQ-2) IN PAIN-RELATED MUSCULOSKELETAL CONDITIONS: A SYSTEMATIC REVIEW}

Joy MacDermid ${ }^{1}$, Samuel Jumbo ${ }^{2}$, Michael Kalu ${ }^{3}$, Tara Packham ${ }^{3}$, George Athwal ${ }^{2}$, Ken Faber ${ }^{2}{ }^{1}$ Western University, Physical Therapy, London, Canada; ${ }^{2}$ Western University, Surgery, London, Canada; ${ }^{3}$ McMaster University, Rehabilitation Science, Hamilton, Canada

Background: The Brief Pain Inventory (BPF-SF) and McGill Pain Questionnaire (SF-MPQ-2) are general-use, self-report, multidimensional pain assessment outcomes frequently used for pain assessment in musculoskeletal (MSK) conditions. Synthesizing knowledge on their measurement properties, as assessed in MSK conditions, should provide a deeper understanding of their strengths and limitations.

Objectives: To systematically locate, critically appraise, compare and summarize clinical measurement research about the BPI-SF and SF-MPQ-2 in pain-related musculoskeletal conditions

Methods: Four databases (Medline, CINAHL, EMBASE \& SCOPUS) were systematically searched for relevant citations, each for the BPI-SF and SF-MPQ-2. We included articles that reported the psychometric properties (e.g. validity, reliability, responsiveness) and interpretability indices (e.g. minimal clinical important difference) of both tools, as assessed in mixed and specific MSK studies. Independently, two reviewers extracted data and assessed the quality of evidence with a structured quality assessment tool for measurement studies and according to the updated COnsensus-based Standards for the selection of health Measurement INstruments (COSMIN) guidelines.

Results: Twenty-five articles were included (BPI-SF, $n=17$; SF-MPQ-2, $\mathrm{n}=8$ ). Both tools lack reporting on their cross-cultural validities and measurement error indices. High quality studies suggest that they are internally consistent $(\alpha=0.83-0.96)$, and they associate modestly with similar outcome measures $(r=0.3-0.69)$. There is evidence that the BPI-SF conforms to its two-dimensional structure in MSK studies; the SF-MPQ-2 four-factor structure was not clearly established. In seven reports, high to moderate quality evidence was seen in supports of the BPI-SF known group validity $(n=2)$ and responsiveness $(n=5)$ but none was available for the SF-MPQ-2. Furthermore, the SF-MPQ-2 was more frequently associated with floor effects in MSK studies than the BPI-SF (SF-MPQ-2, 42\% vs BPI-SF, $6 \%$ ).

Conclusion: The SF-MPQ-2 has emeging evidence whereas the BPI-SF evidence is more mature. Both tools displayed high-quality evidence in support of their internal consistency and criterion-convergent validities. High to moderate quality evidence suggests the BPI-SF subscales have a better responsiveness, retest reliability, known group validity and structural validity than the SF-MPQ-2.

\section{REFERENCES}

[1] Cleeland CS, Ryan KM. Pain assessment: global use of the Brief Pain Inventory. Ann Acad Med Singapore. 1994;23(2):129-138. doi:10.1016/ 0029-7844(94)00457-O.

[2] Dworkin RH, Turk DC, Revicki DA, et al. Development and initial validation of an expanded and revised version of the Short-form McGill Pain Questionnaire (SF-MPQ-2). Pain. 2009;144(1):35-42. doi:10.1016/j. pain.2009.02.007.

[3] Dworkin RH, Turk DC, Trudeau JJ, et al. Validation of the Short-Form McGill Pain Questionnaire-2 (SF-MPQ-2) in Acute Low Back Pain. J Pain. 2015;16(4):357-366. doi:10.1016/j.jpain.2015.01.012.

Disclosure of Interests: None declared

DOI: 10.1136/annrheumdis-2019-eular.3525

\section{AB1329}

THE PRELIMINARY VALIDATION OF LASER DOPPLER FLOWMETRY IN SYSTEMIC SCLEROSIS ACCORDING TO THE OMERACT FILTER: A SYSTEMATIC REVIEW

Karin Melsens ${ }^{1}$, Sarah Van Impe ${ }^{2}$, Carmen Pizzorni ${ }^{3,4}$, Maurizio Cutolo ${ }^{3,4}$, Vanessa Smith ${ }^{1,5} .{ }^{1}$ Ghent University; Ghent University Hospital, Department of Internal Medicine; Department of Rheumatology, Ghent, Belgium; ${ }^{2}$ Ghent University, Ghent, Belgium; ${ }^{3}$ University of Genoa, Research Laboratory and Academic Division of Clinical Rheumatology, Genoa, Italy; ${ }^{4}$ San Martino Polyclinic Hospital, Genoa, Italy, Internal Medicine, Genoa, Italy; ${ }^{5}$ VIB Inflammation Research Center (IRC), Unit for Molecular Immunology and Inflammation, Ghent, Belgium

Background: Systemic sclerosis (SSc) is characterised by a widespread vasculopathy. The vasculopathy comprises the skin microcirculation and results in features such as Raynaud's phenomenon (RP) and digital trofic lesions. The quantification of the skin blood perfusion at the level of the finger (FBP) is a major need in the assessment of SSc in clinical and research setting ${ }^{1}$. Up to now, laser doppler flowmetry (LDF) has been the most thoroughly investigated instrument to assess the FBP in SSc. Objectives: To investigate the validation status of LDF in SSc according to the 'Outcome Measures in Rheumatologic Clinical Trials' (OMERACT) filter.

Methods: Literature was systematically reviewed to detect all reports in which the assessment of the FBP in SSc patients was described. The OMERACT filter, including the domains of truth, discrimination and feasibility was applied and a quality assessment was done by the Good Methods Checklist ${ }^{2}$. Comparison between studies was eased by grouping the results per dynamic test situation (basal circumstances, cold/heat challenge and occlusion).

Results: The systematic search resulted in 4340 hits. After title and abstract screening 228 hits remained and of these, 79 full texts described the assessment by LDF. Fifty studies were included for quality assessment of which 17 studies were retained for conclusion making (fig 1).

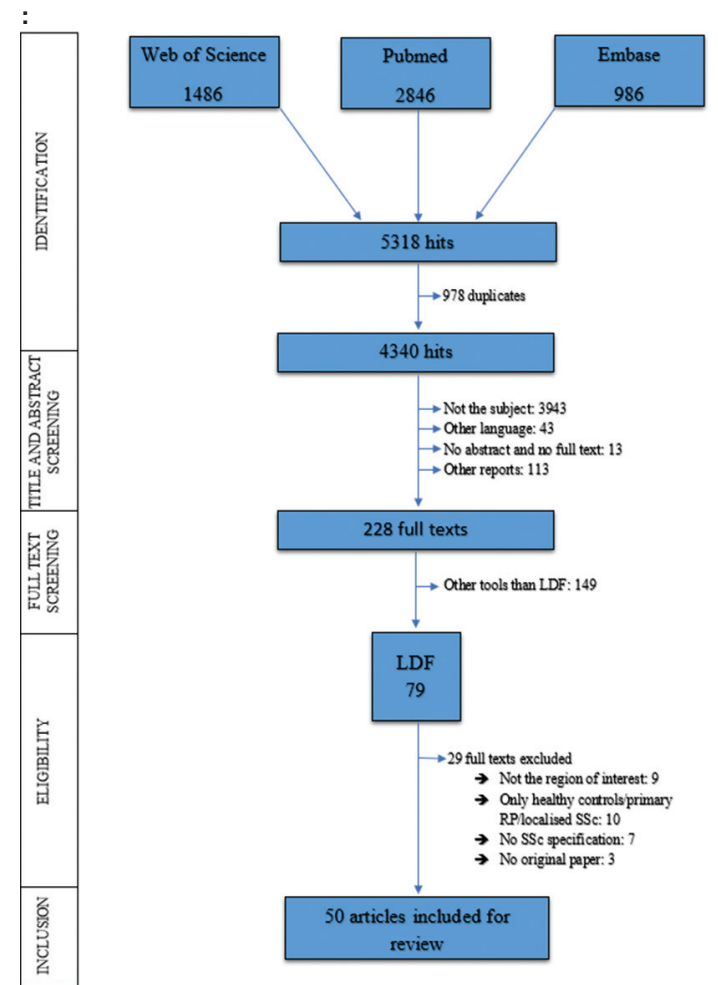

Figure 1. Flowchart of the systematic search

An overview of the validation status of LDF is given in table 1. Expert consensus is lacking on the face and content validity of LDF in SSc. The construct validity of LDF is partially validated (e.g. the correlation with laser speckle contrast imaging was attested in one study). Conflicting results exist on the discriminant capacity of LDF to distinguish healthy from diseased, primary from secondary RP and to differentiate between disease subsets. The addition of a heat challenge, as well as the evaluation of the post-occlusive hyperemic response to the LDF-measurement has the potential to elicit a difference between healthy and diseased. Lastly, there are no data on the feasibility of LDF in SSc. 